
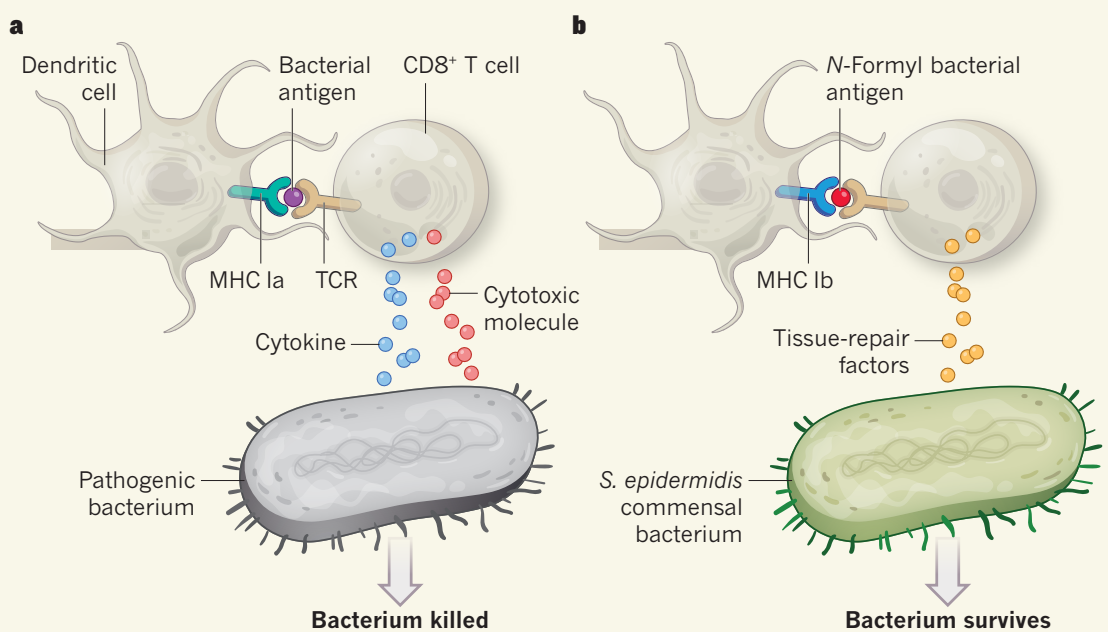

Figure 1 | Response of killer $\mathrm{T}$ cells to bacterial infection. a, An immune cell called a CD8 ${ }^{+} \mathrm{T}$ cell (killer T cell) can help to control infection by a pathogenic bacterium. The T-cell receptor (TCR) recognizes infection when a bacterial peptide called an antigen is presented on dendritic cells of the immune system by an MHC class Ia protein. A conventional T-cell response is then unleashed, and the release of cytokine and cytotoxic molecules triggers inflammation and bacterial destruction. $\mathbf{b}$, Linehan et al. ${ }^{1}$ report an analysis of the killer-T-cell response to Staphylococcus epidermidis using mouse models and human cells. This type of bacterium, called a commensal, can become a long-term, non-pathogenic resident of the body. Killer T cells recognize S. epidermidis when an MHC class Ib molecule presents an antigen containing an $N$-formyl modification of an amino-acid residue. The T cell expresses genes associated with tissue repair, and the bacterium survives this 'unconventional' response. However, such immune responses are probably not neatly divided into these categories, and conventional T-cell responses might in some cases aid tissue repair and unconventional T-cell responses might aid host defence against pathogens.

These and other T-cell populations in humans and mice, such as invariant natural killer T cells and germline-encoded mycolyl-reactive $\mathrm{T}$ cells, also act in response to MHC class Ib-mediated bacterial-peptide recognition ${ }^{6,7}$. A human equivalent to mouse $\mathrm{H} 2-\mathrm{M} 3$ has not been identified so far. It is possible that, if such an equivalent is found, a human T-cell population might fulfil a similar role to that of the $\mathrm{CD} 8^{+} \mathrm{T}$ cells observed by Linehan and colleagues.

As the authors note, many of the day-to-day interactions between immune-system cells and microbes are probably with the commensals in the skin, gut and airways. Therefore, an immune response that maintains the status quo, rather than driving bacterial elimination, which is usually accompanied by inflammation and tissue damage, could be beneficial. But should such a response really be considered unconventional? Although the roles of classical MHC molecules and responding immune cells were identified first, non-classical MHC and MHC-like proteins are evolutionarily older ${ }^{8,9}$ than MHC class Ia molecules. Linehan and

colleagues reveal a direct link between the role of MHC class Ib molecules and tissue homeostasis. Therefore, these functions might have evolved in parallel and represent the broadly 'conventional' functions of the immune system, with additional immune-system specializations evolving over time.

The authors'study hints at the potential broad, long-term effects of microbial residents on the immune system. If future studies mechanistically link individual microorganisms to specific types of immune response, it might be possible not only to understand the roles of some abundant and relatively unexplored immune-cell populations, but also to determine how resident microbes affect health and disease in a way that might lead to new treatment approaches.

Paul Klenerman and Graham Ogg are in the NIHR Biomedical Research Centre, Oxford OX1 3SY, UK. P.K. is also in the Translational Gastroenterology Unit, Nuffield Department of Medicine, University of Oxford. G.O. is also in the MRC Human Immunology Unit, University of Oxford. e-mails:paul.klenerman@ndm.ox.ac.uk; graham.ogg@ndm.ox.ac.uk

1. Linehan, J. L. et al. Cell 172, 784-796 (2018).

2. Sabaté Brescó, M. et al. Front. Microbiol. 8, 1401 (2017).

3. Naik, S. et al. Nature 520, 104-108 (2015).

4. Wang, C.-R. et al. Cell 82, 655-664 (1995).

5. Legoux, F., Salou, M. \& Lantz, O. Annu. Rev. Cell Dev. Biol. 33, 511-535 (2017).

6. Van Rhijn, I. et al. Nature Immunol. 14, 706-713 (2013).

7. Godfrey, D. I., Uldrich, A. P., McCluskey, J., Rossjohn, J. \& Moody, D. B. Nature Immunol. 16, 1114-1123 (2015).

8. Rodgers, J. R. \& Cook, R. G. Nature Rev. Immunol. 5, 459-471 (2005).

9. Goyos, A., Sowa, J., Ohta, Y. \& Robert, J. J. Immunol. 186, 372-381 (2011)

This article was published online on 26 March 2018.

hormones and neuropeptide molecules. After a meal, glucose levels rise, and $\beta$-cells in the pancreas release the hormone insulin. Insulin binds to its receptor on the surface of liver, muscle and fat cells, stimulating them to take up glucose from the bloodstream, and thereby returning blood glucose levels to normal. Conversely, if blood glucose levels drop, for example between meals, pancreatic $\alpha$-cells release the hormone glucagon, which stimulates the liver to break down its reserves of a glucose polymer called glycogen. The release of this glucose into the bloodstream once again returns blood glucose levels to normal.

Disturbance in these interplays can cause severe metabolic disorders, such as diabetes, which results in high blood-glucose levels and a range of deleterious effects. In type I diabetes, $\beta$-cells are destroyed by immune cells, and insulin is not produced. In type II diabetes, insulin is produced, but its levels are too low

. On page 647, Riddle et al. ${ }^{1}$ reveal the surprising way in which cavefish regulate their glucose metabolism, a finding that might have implications for our understanding of human diabetes.

Blood-glucose regulation is essential for the body to function normally. In humans, this is achieved by a complex network of interactions between several organs that is mediated by 


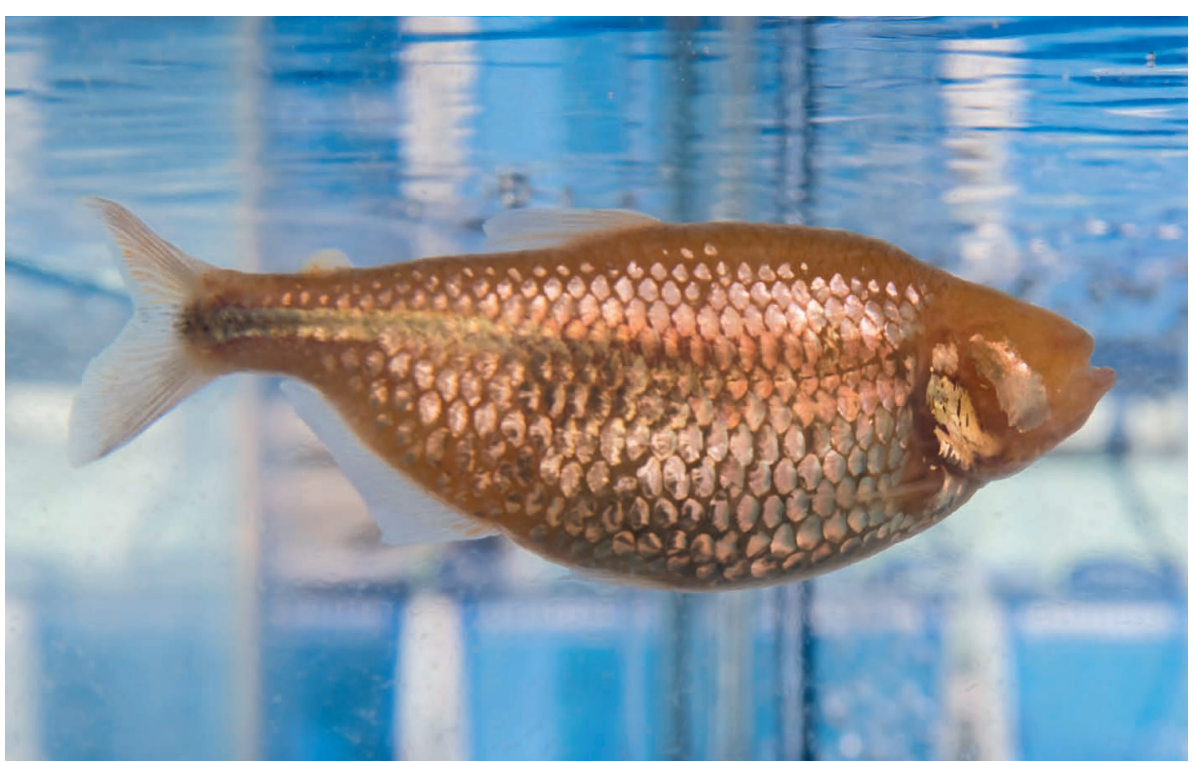

Figure 1 | A laboratory-raised blind Mexican cavefish (Astyanax mexicanus) from the Tinaja cave population. Riddle et al. ${ }^{1}$ report that cavefish from the Tinaja and Pachón caves in Mexico have high blood-glucose levels owing to a mutation in the insulin receptor protein that is identical to one that causes diabetes and a range of health problems in humans $s^{5,6}$. Surprisingly, these cavefish are healthy and have a normal lifespan.

or it is not recognized by the target tissues. This lack of an insulin response is known as insulin resistance.

Although studies of diabetes have focused mainly on mammalian systems, other creatures might offer a fresh perspective on the condition. Cavefish certainly look out of the ordinary, with their depigmented pink skin and eyeless faces (Fig. 1). Hints that these fish have unusual metabolic adaptations for coping with their lightless and seemingly food-poor habitat led Riddle and colleagues to investigate them further. Cavefish have evolved behavioural and physiological traits that differ from those of the river-dwelling members of their species. Compared with river dwellers, cavefish are fatter, owing to a larger lipid reserve; they are better able to cope with starvation ${ }^{2}$; have a lower oxygen consumption and metabolic rate $^{3}$; and the absence of circadian rhythms in their metabolism enables them to make a $27 \%$ saving in energy usage ${ }^{4}$.

Riddle and colleagues now reveal another metabolic surprise. Cavefish have higher blood glucose levels than the river dwellers of the species. The authors' investigation revealed that cavefish are insulin resistant and have a mutant version of the insulin receptor, encoded by the insra gene, that does not bind and transduce the insulin signal. This mutation, in which the usual amino-acid residue proline is replaced by leucine, is identical to a change that causes diabetes and health problems in humans ${ }^{5,6}$.

To investigate the effect of this mutation in the fish, the authors used the CRISPRCas gene-editing technique to generate zebrafish (Danio rerio) carrying mutated insra. This experiment would perhaps have been even more informative if performed in a river-dwelling $A$. mexicanus, but the results are clear: if both the maternally and paternally inherited copy of insra have the mutation, the fish are insulin resistant and overweight. The insulin resistance makes sense, given what is known about this mutation in humans, but the weight gain is puzzling and unexpected, because insulin is a growth hormone. In humans and rodents, lack of a functional insulin receptor is associated with growth retardation and low body fat $^{7}$.

Does this mean that insulin signalling has divergent roles in fish and mammals? Zebrafish and A. mexicanus cavefish and river dwellers have two versions of the insulin receptor gene, called insra and insrb. This raises the question of why the receptor encoded by insrb doesn't compensate for the non-functional receptor encoded by the mutant version of insra. In zebrafish, the two receptors have overlapping roles in growth control and glucose metabolism through the action of the insulin signalling pathway, but they have opposing effects on lipid metabolism, and the receptor encoded by insra promotes lipid synthesis ${ }^{8,9}$. However, in cavefish and in the gene-edited zebrafish, the insra gene is mutated, making this weight gain even more puzzling.

Perhaps one of the strangest findings is that cavefish have a respectable lifespan of more than 14 years, which is similar to that of the river-dwelling form. Moreover, they show fewer signs of age-related tissue decline. Elevated blood-glucose levels can damage tissue if the glucose bonds with proteins in a process termed glycation, which is linked to a range of health problems in people who have diabetes. However, cavefish do not have high levels of glycated proteins, so they must have evolved a compensatory mechanism. One way to unmask this mechanism might be to study cavefish in which gene editing is used to correct the insra gene mutation. The authors will undoubtedly be working on this, given the potential biomedical importance of identifying a glycation-inhibiting mechanism.

Further genetic analysis by the authors reveals the complexity of the mechanism that underlies the elevated-glucose phenomenon. The beauty of studying $A$. mexicanus is that the cavefish and river dwellers can interbreed. The authors therefore studied the patterns of inheritance of the elevated-glucose trait, and found that insra is not the only gene involved. In the offspring of interbreeding crosses, all of the fish that had high blood glucose had the cavefish insra mutation. However, some of the fish with this mutation did not have high blood glucose, indicating that the insra mutation is necessary but not sufficient to alter blood glucose regulation. The identification of other genes involved might shed light on the weight-gain phenomenon.

About 30 caves in Mexico are home to cavefish colonies ${ }^{10}$. Some of these populations arose from independent colonization events by river-dwelling ancestors. The authors compared three cavefish populations - two from the Tinaja and Pachón caves, which are in the same mountain range, and the third from the Molino cave in another mountain range. The authors found that all of these populations have high blood-glucose levels, but only those from Tinaja and Pachón have the insra mutation. Another mechanism must be at work in fish from Molino. The authors' analyses suggest that the insulin signalling pathway is unaffected in these fish, which also have high levels of glycated proteins. Molino cavefish have lived for more than 13 years in our laboratory, suggesting that they also cope well with their unusual metabolism.

The convergent evolution of this extraordinary blood-glucose dysregulation, through different mechanisms in fish from different caves, seems counter-intuitive in terms of species fitness. What compensates for this surprising evolutionary path? The authors suggest that high fat reserves might help the fish to survive during extended periods of food deprivation.

However, perhaps something else is at play. Caves are probably not as food-poor as they seem. In the Tinaja cave (home of the main population investigated by Riddle and colleagues), the cavefish feed on mud that has a high carbon content ${ }^{11}$. Moreover, a substantial proportion of the fish in the Tinaja and Pachón populations have only one copy of the mutant version of insra. If a beneficial mutation has not spread throughout an entire population, one possible interpretation is that the mutation arose relatively recently in the population's history. This would imply that the early cave settlers did not have the benefit of being 
fat and starvation-resistant. Exciting times lie ahead for explorations of the metabolic and adaptive changes that occurred as different cavefish populations evolved, and such studies might uncover the underlying evolutionary forces responsible for this striking metabolic adaptation.

Sylvie Rétaux is at the Paris-Saclay Institute of Neuroscience, University of Paris-Sud and University of Paris-Saclay, Gif-sur-Yvette 91198, France. e-mail:retaux@inaf.cnrs-gif.fr

1. Riddle, M. R. et al. Nature 555, 647-651 (2018).

2. Aspiras, A. C., Rohner, N., Martineau, B.,
Borowsky, R. L. \& Tabin, C. J. Proc. Natl Acad. Sci. USA 112, 9668-9673 (2015)

3. Hüppop, K. Environ. Biol. Fishes 17, 229-308 (1986).

4. Moran, D., Softley, R. \& Warrant, E. J. PLoS ONE 9 , e107877 (2014).

5. Atray, A. et al. J. Diabetol. 4 (2), 2 (2013).

6. Carrera, P. et al. Hum. Mol. Genet. 2, 1437-1441 (1993).

7. Savage, D. B. \& Semple, R. K. Curr. Opin. Lipidol. 21 , 329-336 (2010).

8. Toyoshima, Y. et al. Endocrinology 149, 5996-6005 (2008)

9. Yang, B.-Y. et al. Am. J. Physiol. Endocrinol. Metab. http://dx.doi.org/10.1152/ajpendo.00227.2017 (2017).

10.Mitchell, R. W., Russell, W. H. \& Elliott, W. R. Spec. Publ. Mus. Texas Tech. Univ. 12 (1977).

11.Simon, V. et al. EvoDevo 8, 23 (2017).

This article was published online on 21 March 2018.

\section{The tornadoes of sudden cardiac arrest}

A clever combination of techniques has enabled, for the first time, simultaneous visualization of the 3D waves of electrical and mechanical activity that are responsible for many cases of sudden cardiac death. SEE LETTER P.667

\section{JOSÉ JALIFE}

$\mathrm{S}$ udden cardiac arrest is a common cause of death in people with coronary artery disease $^{1}$, and also kills many young people who have heritable heart diseases. In both cases, sudden death often occurs because of a heart-rhythm defect called ventricular fibrillation ${ }^{2,3}$, in which the heart rate increases dramatically and cardiac-muscle contractions in the heart's ventricular chambers become uncoordinated, rendering the heart unable to pump blood. Blood pressure decreases, leading to unconsciousness, and death follows unless a defibrillating electrical shock is applied. The mechanism underlying this anomaly has been debated for more than a century. On page 667, Christoph et al. ${ }^{4}$ show that the use of panoramic fluorescence imaging with a voltage-sensitive dye, combined with ultrasound imaging, could considerably advance our understanding of ventricular fibrillation. The study has potential implications for a broad range of researchers, from physicists to cardiologists.

Normal heart rhythm is maintained by cyclic changes in the electrical currents that drive and coordinate heart-muscle contraction. But during ventricular fibrillation, electrical impulses stop tracking their normal paths across the heart, and instead adopt a complex, vortex-like pattern similar to eddies in water or a tornado in the atmosphere. Fluorescence imaging of the voltage on the heart's surfaces has revealed ${ }^{5}$ that these electrical vortices involve highly periodic, spiralling waves of activity organized around a central point called a rotor. The rotor, which is the organizing centre of fibrillation ${ }^{6}$, spins rapidly and can meander across the heart's surface, generating turbulent, wave-like behaviour.

Until now, fluorescence imaging has allowed the visualization of rotors only on the surfaces of the heart's chambers, and so activity inside the ventricles has been inferred through computer simulations ${ }^{7}$. The $3 \mathrm{D}$ equivalent of $2 \mathrm{D}$ spiral waves (called scroll waves) and the filament-shaped rotors at their centres (vortex filaments) could only be reconstructed using simultaneous $2 \mathrm{D}$ video images of spiral waves on the outer and inner surfaces of the heart ${ }^{8}$. This is problematic, because it has not been possible to prove that the electromechanical changes that occur in the heart proper during ventricular fibrillation match predictions made by simulations. For example, cardiac defibrillation by an electrical shock is the only known treatment for ventricular fibrillation, but our understanding of how it works is currently based on numerical predictions.

Christoph et al. have overcome this hurdle, thanks to a clever combination of techniques. The authors kept isolated pig and rabbit hearts alive by perfusion with a warm solution through the coronary arteries. They induced ventricular fibrillation in the hearts, and used highresolution $4 \mathrm{D}$ ultrasound imaging to document changing mechanical strain over time.

The group then combined these data with more-conventional 2D fluorescence imaging,

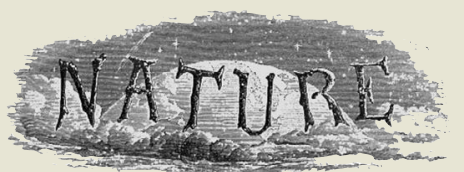

50 Years Ago

Throughout this week an unusual meeting has been taking place near Paris ... experts from all over France - and some from other parts of the world - have been discussing what the world will be like in the year 2020. To keep the discussion within sensible limits, it has been set in a geographical context so that each day has been spent discussing the way in which different technological developments will affect the way land is used. It is clear that this meeting ... is intended to be the first of a series which could, no doubt, last long enough for the results of this meeting to be compared with the reality of 2020 .

From Nature 30 March 1968

100 Years Ago

Another Indian "miracle" has been explained by scientific investigation. The Pioneer Mail of January 11 reports a lecture by Sir J. C. Bose on "The Praying Palm Tree" of Faridpur. While the temple bells call the people to evening prayer, this tree has recently been seen to bow down in prostration, and to erect its head on the following morning. Large numbers of pilgrims have been attracted to the place, and offerings to the tree are said to have been the means of effecting marvellous cures. Sir J. C. Bose first procured photographs which proved the phenomenon to be real. The next step was to devise a special apparatus to record continuously the movement of the tree by day and night. The records showed that it fell with the rise of temperature and rose with the fall. The records obtained in the case of other trees brought out the fact that all the trees are moving, each movement being due to changes in their environment. From Nature 28 March 1918 